The peak longitudinal strain in patients correlated with the $\mathrm{RV}$ end diastolic volume index $(\mathrm{r}=0.38$, p 0.03), RV end systolic volume index $(\mathrm{r}=0.52, \mathrm{p} 0.002), \mathrm{RV}$ EF $(\mathrm{r}=0.535$, $\mathrm{p}$ 0.002) and RV mass index $(\mathrm{r}=0.43, \mathrm{p}$ 0.01). The mid RV circumferential strain only correlated with RV EF $(r=0.40, p$ 0.02 ) and no other remodelling parameters.

Discussion RV longitudinal strain was reduced in patients with a systemic RV irrespective of whether the ventricular configuration was single or dual. No difference was seen between the single circulation systemic RV compared to the dual circulation systemic RV, suggesting that RV remodelling is mostly in response to the systemic position rather than the ventricular configuration. Longitudinal studies will be required to assess the utility of the longitudinal strain in the prediction of outcomes in the follow up of these patients.

\section{AN ASSESSMENT OF THE PERFORMANCE OF THREE BIOLOGICAL TRICUSPID VALVE REPLACEMENTS IN PATIENTS WITH CONGENITAL HEART DISEASE}

Thomas Fleck, Paul Clift*. Queen Elizabeth Hospital

10.1136/heartjnl-2017-311726.83

Objective To assess the performance of three Biological Tricuspid Valve replacements (St Jude Epic, Perimount and Hancock) in congenital heart disease patients. Currently, there is little comparative data on the long term performance of these biological tricuspid valve replacements.

Methods We audited the performance of the valve replacements in all the patients with Congenital Heart Disease $(n=50)$ who had Biological Tricuspid Valve replacements in the Queen Elizabeth Hospital, Birmingham, from 2000 to present. For each patient, we collected echocardiogram data at baseline (the first data available after their surgery) and at their most recent review. Valve performance was assessed according to criteria set out by the American Society of Echocardiology (ASE) in terms of valve stenosis and regurgitation. We also looked at patient-reported symptoms before surgery and at most recent review.

Valve was Stenosed if one or more of the following was met: Mean gradient $6 \mathrm{mmHg}$; Peak velocity $>1.7 \mathrm{~m} / \mathrm{s}$; Pressure half time $>230 \mathrm{~ms}$.

Valve was Regurgitant if the following was met: Tricuspid regurgitation $>$ Mild regurgitation on report.

Patient Declined symptomatically if one or more of the following was met: NYHA class unchanged or worsened; new arrhythmia post-op; decrease in measured peak $\mathrm{VO}_{2}$. All mortalities that occured during follow-up were recorded. These patients were not included in the analysis.

Results Only two Hancock valves had been used since 2000, which was too few to be able to draw any reliable conclusions. Table 1 presents background data for the other two valves; Table 2 presents the comparison of their performance. Proportionately fewer Perimount valves became stenosed or regurgitant than the St Jude Epic valves. St Jude Epic valves were associated with improved peak $\mathrm{VO}_{2}$, however average NYHA class reduction (improvement) was greater in those with the Perimount. This may reflect the case mix in this small number of patients.

\begin{tabular}{lll} 
Abstract 84 Table 1 & & \\
\hline & Perimount Valve & St Jude Epic Valve \\
\hline No. of operations performed & 16 & 27 \\
No. of survivors & 13 & 24 \\
Average follow-up (years) & 3.49 & 3.00 \\
Total patient-years of follow-up & 41.9 & 68.9 \\
\hline
\end{tabular}

\section{Abstract 84 Table 2}

\begin{tabular}{lll}
\hline & Perimount Valve & St Jude Epic Valve \\
\hline Percentage 'stenosed' at baseline & 0.0 & 8.7 \\
Percentage 'stenosed' at follow-up & 11.1 & 22.2 \\
Percentage difference & 11.1 & 13.5 \\
Percentage 'regurgitant' at baseline & 9.1 & 0.0 \\
Percentage 'regurgitant' at follow-up & 23.1 & 21.7 \\
Percentage difference & 14.0 & 21.7 \\
Percentage 'declined symptomatically' & 46.2 & 37.5 \\
Average NHYA class change & -1.31 & -0.91 \\
Average V02 max change & -8.6 & 5.43 \\
\hline
\end{tabular}

Discussion and conclusion Tricuspid valve replacement is rarely performed and the choice of which valve to use is not assisted by any published data. This audit suggests that the Perimount valve in the tricuspid position has a marginally better echo profile than the St Jude Epic valve over a three year follow up. The cohort is too small to make recommendations on which valve to use, but has highlighted a relatively high early attrition rate in terms of bioprosthetic valve function in the tricuspid position.

Therefore we propose that valve surveillance is carried out annually for these valves and that valve dysfunction merits further investigation. We propose to perform CT evaluation of dysfunctional bioprosthetic valves for evidence of thrombus and this will be prospectively audited.

\section{POST OPERATIVE COMPLICATIONS IN ADULT CONGENITAL HEART DISEASE}

Emma Pick, Jo Quirk*, Jo Birkett, Damien Cullington, James Oliver, Stefano Congiu, Osama Jaber, Carin Van Doorn, Kate English. Leeds Teaching Hospitals Trust

\subsection{6/heartjnl-2017-311726.84}

Introduction Increasing numbers of adults with congenital heart disease (ACHD) are undergoing redo or primary surgical procedures. There is little available data relating to post-operative mortality and morbidity in this group.

Methods We examined 99 consecutive ACHD patients who underwent cardiac surgery in our institution over twelve months from April 2015. Data collected included age, body mass index (BMI), type of procedure, whether the procedure was a primary procedure or a redo, length of stay and the occurrence of any complications. Complications were classified as requiring intervention or self-limiting. Whether complications had permanent long term sequelae or not was assessed.

Results Patients were aged between 16 and 69 years. 41 patients underwent a primary procedure, and there were 58 redo operations. 19 different primary procedures were 
performed, and 20 patients underwent a multi-faceted procedure. $56 \%$ of patients had a BMI above the normal range. Median length of stay was 8 days, IQR 7-10, range 3-42.

68 complications occurred in 46 patients. There were 2 deaths. There were 54 complications requiring intervention, and 14 self-limiting complications. 28 patients had 1 complication, 14 patients had 2 complications and 4 patients had 3 complications. The most common complications requiring intervention were post-operative chest infection requiring oral or IV antibiotics $(n=19)$, atrial arrhythmia requiring antiarrhythmic therapy or cardioversion $(n=7)$, post-operative wound infection requiring IV or oral antibiotics $(n=7)$, and pericardial effusion requiring drainage $(n=5)$. Self-limiting complications included 1 patient with nodal rhythm with pauses, 2 patients with an acute confusional state, 1 patient with a groin haematoma (following fem-fem bypass), 1 patient with a residual VSD following resection of double chambered right ventricle and 1 patient with gastroenteritis.

Apart from the 2 deaths, there were 4 patients who had long term sequelae from their complication. 1 patient in their 60 's suffered a peri-operative cerebellar stroke, 1 patient required a permanent pacemaker, and 2 patients had vocal cord palsy leading to hoarse voice.

Post-operative complications increased in incidence with both increasing age and increasing BMI. (Figs 1\&2)

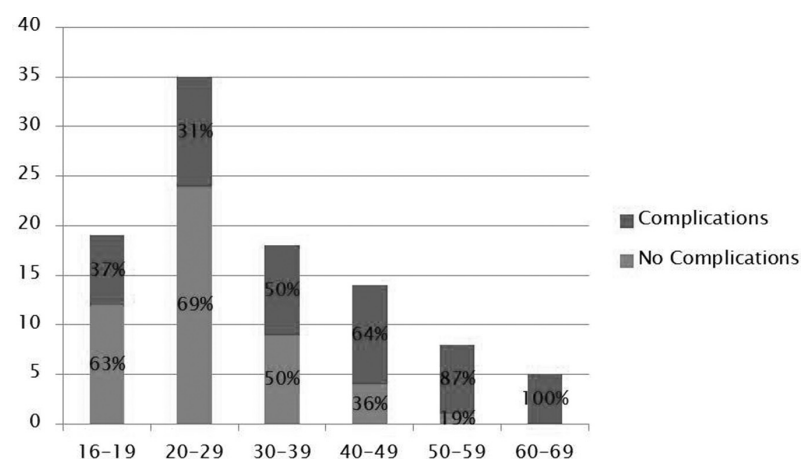

Abstract 85 Figure 1 The incidence of post-operative complications according to patient age.

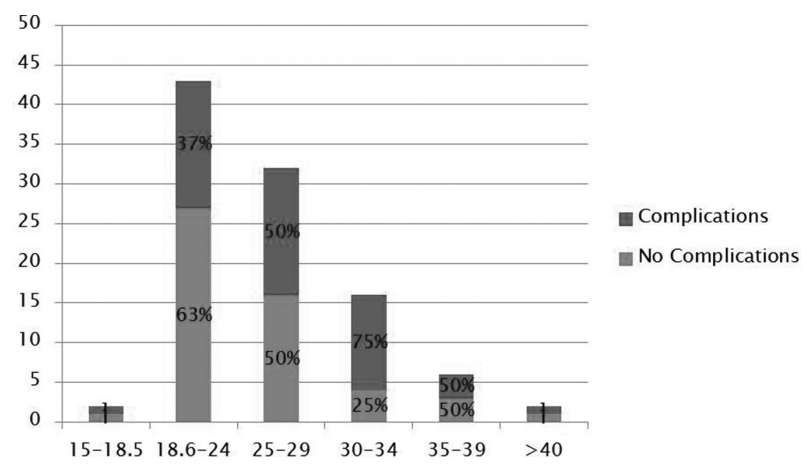

Abstract 85 Figure 2 The incidence of post-operative complications according to patient BMI.
Conclusions Increasing numbers of cardiac surgical procedures are being performed in ACHD patients because of their increasing numbers and complexity. This study demonstrates that even complex surgery can be undertaken at low mortality risk. Just under $50 \%$ of patients experience one or more perioperative complications but the vast majority are successfully treated with no long term adverse consequences. Complications appear to increase with advancing age and BMI.

\section{Stable IHD/Prevention/Hypertension/Lipids}

\section{ACHIEVEMENT OF BLOOD PRESSURE, LIPIDS AND GLUCOSE TARGETS IN PATIENTS WITH CORONARY DISEASE OR AT HIGH RISK OF DEVELOPING ATHEROSCLEROTIC CARDIOVASCULAR DISEASE IN EUROPE: RESULTS FROM EUROASPIRE SURVEY}

${ }^{1}$ Kornelia Kotseva*, ${ }^{1}$ David Wood, ${ }^{2}$ Catriona Jennings, ${ }^{3}$ Dirk De Bacquer, ${ }^{3}$ Guy De Backer. ${ }^{1}$ Imperial College London; ${ }^{2}$ Imerial College London; ${ }^{3}$ University of Ghent

\subsection{6/heartjnl-2017-311726.85}

Introduction EUROASPIRE IV is a cross-sectional survey undertaken in 26 European countries to evaluate the implementation of the Joint European Societies guidelines on cardiovascular (CVD) prevention in everyday clinical practice.

Methods Patients $<80$ years with 1 ) coronary disease or 2) identified as high CVD risk on the basis of being on blood pressure and/or lipid-lowering medication and/or having diabetes, were interviewed at least six months after the recruiting coronary event or the start of medication.using standardised methods and instruments.

Results A total of 7998 coronary patients (24\% females) and 4579 high CVD risk individuals $(59 \%$ women) were interviewed. The risk factor control was poor in both groups. Among those on blood pressure lowering medication the target of $<140 / 90 / 90 \mathrm{~mm} \mathrm{Hg}(<140 / 80 / 80 \mathrm{~mm} \mathrm{Hg}$ in people with diabetes) was achieved by $53 \%$ of coronary patients and $43 \%$ of high CVD risk individuals. Only $21 \%$ of coronary patients attained the LDL-cholesterol goal of $<1.8 \mathrm{mmol} / \mathrm{L}$ and $33 \%$ of high CVD risk individuals achieved the target of $<2.5 \mathrm{mmol} / \mathrm{L}$. Among people treated for type 2 diabetes mellitus, $53 \%$ of coronary patients and $59 \%$ of high CVD risk individuals achieved the glycated haemoglobin (HbA1c) target of $<7.0 \%$. Compared with data from previous surveys the control of blood pressure and LDL-cholesterol in coronary patients improved significantly. There was a trend in improving of blood pressure and lipids management in patients at high CVD risk. In both groups, glycaemic control in patients with previously known diabetes was similar to that observed in the third survey.

Conclusion Despite advances in patient management there is further potential to improve risk factor control in patients with coronary disease and those at high risk of developing CVD. Although the risk factors studied were better controlled than in previous surveys, there is still considerable potential to further reduce cardiovascular risk through lifestyle and optimised cardioprotective medications. A significant gap continues to exist between the prevention guidelines and their adherence in everyday clinical practice in Europe. 